\title{
EDITORIAL
}

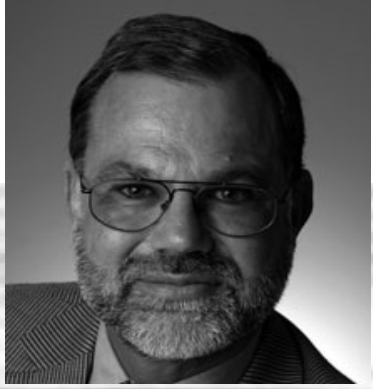

Prof. Dr. Jörg Haake

FernUniversität in Hagen

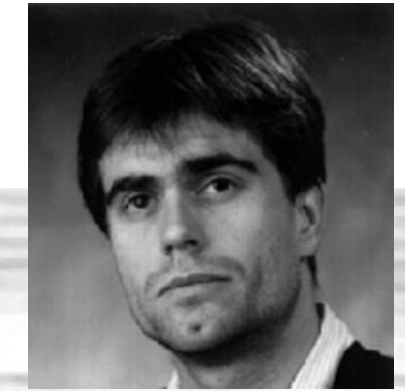

Prof. Dr. Volker Wulf Universität Siegen

\section{Kontextbasierte Interaktion}

Anwendungen der Informatik werden zunehmend mobil und allgegenwärtig, nutzen neue Interaktionsmodalitäten, und unterstützen in immer stärkerem Maße die Kooperation zwischen Menschen. Dabei beobachten wir, dass die Interaktion sowohl zwischen Benutzer und Anwendung als auch zwischen den Nutzern je nach Kontext stark variiert. Eine an Vielfältigkeit und technischer Leistungsfähigkeit gewinnende Sensorik ermöglicht es, Aspekte des Nutzungskontexts automatisch zu erfassen und darauf aufbauende Funktionalität und Interaktionsformen zu entwickeln. Allerdings zeigen empirische Befunde und theoretische Reflexionen, dass eine mechanistische Interpretation des Nutzungskontextes, die automatisiert nach demselben Algorithmus abläuft, der Differenziertheit individueller und kollektiver Handlungspraktiken und deren Weiterentwicklung im zeitlichen Verlauf kaum gerecht wird. Wenn aber ein ausschließlich auf Automatismen setzender Umgang mit dem Nutzungskontext sich als problematisch erweist, dann ist zu untersuchen, wie Nutzer technisch dabei unterstützt werden können, den für sie jeweils relevanten Nutzungskontext zu konstruieren. Es stellt sich damit die Frage nach einer geeigneten technischen Gestaltung und sozialen Einbettung kontextadaptiver Interaktion.

Das vorliegende Sonderheft befasst sich insbesondere mit Ansätzen für kontextbasierte Interaktion zur Unterstützung schwach strukturierter wissensintensiver Arbeit. Neben anderen Kontextdimensionen können hier insbesondere auch Zustände gemeinsamer Aktivitäten und kollektiv genutzter Artefakte benutzt werden, um Anpassungen des Systemverhaltens bzw. der Interaktion zwischen den Benutzern anzustoßen. Die sechs Beiträge des Sonderhefts befassen sich mit unterschiedlichen Aspekten und Anwendungen kontextbasierter Interaktion.

Die ersten beiden Beiträge befassen sich mit kontextbasierter Adaption in Kooperationsumgebungen: Veiel, Lukosch und Haake beschreiben einen Ansatz für die kontinuierliche Anpassung der Dienste und Interaktionsmöglichkeiten eines gemeinsamen Arbeitsbereichs an wechselnde Kooperationskontexte bzw. Anforderungen eines Teams. Adaption der Benutzungsoberfläche findet hier auf Basis eines Frameworks für Kontextmodellierung und expliziter Policies zur kontextbasierten Adaption des gemeinsamen Arbeitsbereichs statt. Tom Gross erweitert die Betrachtung der Kontextadaptivität auf ubiquitäre Arbeitsumgebungen. In seinem Beitrag stellt er sowohl Anforderungen und Ansätze zur Erkennung von Benutzer- und Kooperationsanforderungen als auch kooperative und ubiquitäre Basistechnologien vor.

Die nächsten drei Beiträge adressieren die Generierung und Nutzung kontextabhängiger Empfehlungen zur Unterstützung schwach strukturierter wissensintensiver Arbeit. Hussein und Ziegler schlagen mit Context Views einen neuartigen perspektivenorientierten Ansatz zur Definition von Kontext in Empfehlungssystemen vor. In dessen Implementierung präsentieren sie ein Framework zur kontextabhängigen Integration bei der Empfehlungsgenerierung auf einer Menge digitaler Ressourcen. Koch und Ott stellen das Konzept der CommunityMirrors vor. Sie nutzen große Wandbildschirme an halböffentlichen Orten, um für kooperative Wissensprozesse kontextabhängig unbekannte, aber sozial relevante, Information verfügbar zu machen. Schließlich beschäftigen sich Ley, Betz, Pipek und Wulf mit der Koexistenz von physikalischen und virtuellen Artefakten bei wissensintensiver Arbeit und Möglichkeiten zu deren besseren Verknüpfung. Am Anwendungsfall wissenschaftlicher Arbeit in der angewandten Informatik untersuchen sie, wie der Umgang mit physischen Wissensartefakten, hier Büchern, mittels RFID-Technologie nachvollzogen 


\section{$\{$ EDITORIAL}

werden kann und wie diese mit ihren digitalen Repräsentationen verbunden werden können. Die so entstehenden dichten intermedialen Nutzungshistorien können sowohl für die kontext-spezifische Generierung von Literaturempfehlungen als auch zur Identifikation von Wissensträgern genutzt werden.

Klamma, Jarke und Renzel betrachten in ihrem Beitrag kontextbasierte Interaktion als Herausforderung des Requirements Engineerings. Sie interessieren sich für emergente virtuelle Gemeinschaften und für Möglichkeiten, deren kontext-spezifischen Unterstützungsbedarfe mittels eines integrierten Systemgestaltungsansatzes für Community Software zu erfassen. Sie präsentieren dazu einen Dienstebaukasten, der in die von der Community genutzten Informationssysteme integriert werden kann.

Abschließend setzt sich das aktuell Schlagwort "Sozio-Informatik" auf einer etwas grundsätzlicheren Ebene mit dem sich verändernden Charakter der Angewandten Informatik auseinander. Durch die zunehmende Durchdringung der Lebenswelt mit IT-Artefakten, bemisst sich die Güte informatischen Schaffens neben technischen Kriterien zunehmend auch an der Art wie diese Artefakte mit den sie anwendenden sozialen Systemen interagieren, welche neuartigen Handlungs- und Entwicklungsmöglichkeiten sie schaffen. Als Fallstudien zur Veranschaulichung eines sozio-informatischen Forschungsansatzes wird die Entwicklung von Landmarken, einer innovativen indoor-Navigationsunterstützung für Feuerwehrleute in brennenden Gebäuden, vorgestellt.

Das Sonderheft entstand auf Initiative des Forschungsverbunds "Kontextadaptive Interaktion in kooperativen Wissensprozessen", der aus Mitteln der Deutschen Forschungsgemeinschaft (DFG) gefördert wird. Wir bedanken uns bei den Gutachtern der hier abgedruckten Beiträge, insbesondere bei Horst Oberquelle, Universität Hamburg, Dirk Riehle, Universität Nürnberg-Erlangen, Bernt Schiele, TU Darmstadt, Johann Schlichter, TU München, Albrecht Schmidt, Universität Stuttgart und Ralf Steinmetz, TU Darmstadt, für ihre kritischen und konstruktiven Anregungen. Wir danken dem Medienkünstler Michael Rodemer, University of Michigan, Ann Arbor, für die Gestaltung des Einbandes zum Sonderheft.

Wir wünschen viel Spaß beim Lesen und freuen uns auf Ihr Feedback.

\section{Jörg M. Haake, Volker Wulf}

